\title{
Nonlinear Fault Detection for Hydraulic Systems
}

\author{
Martin L. Leuschen ${ }^{1}$, Ian D. Walker ${ }^{2}$, and Joseph R. Cavallaro ${ }^{1}$
}

\begin{abstract}
One of the most important areas in the robotics industry is the development of robots capable of working in hazardous environments. As humans cannot safely or cheaply work in these environments, providing a high level of robotic functionality is important. Our work in this area focuses on a fault detection method known as analytical redundancy, or AR. In this paper we discuss the application to a hydraulic servovalve system of our novel rigorous nonlinear AR technique. AR is a model-based state-space technique that is theoretically guaranteed to derive the maximum number of independent tests of the consistency of sensor data with the system model and past control inputs. Conventional linear AR is only valid for linear sampled data systems. However, our new nonlinear AR (NLAR) technique maintains traditional linear AR's mathematical guarantee to generate the maximum possible number of independent tests in the nonlinear domain. Thus NLAR allows us to gain the benefits of AR testing for nonlinear systems with both continuous and sampled data.
\end{abstract}

\section{Introduction}

The usefulness of robots in hazardous situations is highly dependent on their reliability [8-10,30]. Chemicals and radiation can damage robotic components, and many environments can be made more hazardous by actions of a malfunctioning robot. As humans usually cannot enter hazardous environments to repair or remove a failed robot, such failures can be very dangerous and costly. Thus, our team has investigated reliability issues for robots extensively [17-19,23]. A fault detection method known as analytical redundancy $[4,16]$, or AR, is the focus of this particular paper. AR is a model-based technique that derives the maximum number of independent tests from the state-space control model of the system. These AR tests monitor the consistency of sensor data with the linearized system model and past control inputs. The tests determine whether the system is performing nominally, or is deviating from the desired plan and presumably under fault conditions. Our group has used the linear version of the AR technique successfully on electrical robotic systems in the past [30], and has also applied our nonlinear version to nonlinear hydraulic systems such as the Rosie robot discussed below.

In previous papers $[19,23]$, we have discussed the derivation through linear AR of a suite of model based tests for the default sensor package for hydraulic wheel actuators, and introduced an approximate technique for using AR efficiently in nonlinear systems. Some of these tests are comparison of the actual 
system response to control inputs to the predicted response indicated by the model. These show that AR clearly monitors known behaviors of the system. The other tests uncovered by the linear AR analysis reflect higher order state interdependencies, as discussed later and in [27]. These tests give us important additional information about the system that we might not have normally examined.

These previous tests are all based at least partially on linear models of the system. However, the hydraulic valve and motor system behavior is highly nonlinear in nature, which leads to a degradation in the performance of the AR method. Linear AR can miss or improperly detect faults in this situation, so nonlinear AR techniques are highly desirable. We now show our results for rigorously extending the linear AR tests into the nonlinear realm.

\section{Nonlinear Analytical Redundancy}

Let us begin by defining a nonlinear state-space system with states, inputs, and outputs.

$$
\begin{aligned}
& \underline{\dot{x}}(t)=\underline{f}(\underline{x}(t))+\sum_{i} \underline{g}_{i}(\underline{x}(t)) \cdot \underline{u}(t) \\
& \underline{y}(t)=\underline{h}(\underline{x}(t))
\end{aligned}
$$

The corresponding linear system model is:

$$
\begin{aligned}
& \dot{\dot{x}}(t)=A \underline{x}(t)+B \underline{u}(t) \\
& \underline{y}(t)=C \underline{x}(t)
\end{aligned}
$$

NLAR uses the left null-matrix of the observability:

$$
[\Omega(\underline{f}, \underline{g})]\left[\mathcal{O}_{\triangle}(\underline{f}, \underline{g})\right]=\underline{0}
$$

where the model-based observability $\mathcal{O}_{\triangle}(f, \underline{g})$ is calculated using standard methods using the model equations for linear systems, and our modified 'triangular' method $[16,21]$ for nonlinear systems. The $\triangle$ refers to this method, which reduces to the standard method in the linear case) The linear observation space is the rows of the matrix $\left[C, C A, C A^{2} \ldots\right]$. For nonlinear systems, the Lie Derivative operation $L_{f} h_{i}$ is used to combine the vector functions $\underline{f}, \underline{g}_{i}$ with elements of $\underline{h}$, and the observation space is generated from appropriate combinations of $L_{\underline{k}} h_{i}, k \in\left\{\underline{f}, \underline{g}_{1}, \underline{g}_{2}, \ldots\right\}$. For more details, see $[15,16,21,22]$.

However, if the system is observable it is also possible to express the observability in terms of sensor readings $\underline{y}$ and control inputs $\underline{u}[15,16,22]$ in addition to the state and model. This observability will be referred to as the 'dynamically derived observability,' or $\mathcal{O}_{\triangle D D}(\underline{y}, \underline{u})$. The important aspect of this formulation is the explicit dependence of every element of it on the inputoutput behavior of the system as it is functioning at the time the observability 
is calculated. By taking the product of $\mathcal{O}_{\triangle D D}(y, \underline{u})$ with the left null-matrix from equation 3 we can generate the suite of NLAR residual tests $\underline{R}$ :

$$
[\Omega(\underline{f}, \underline{g})]\left[\mathcal{O}_{\triangle D D}(\underline{y}, \underline{u})\right]=\underline{R}=[0]
$$

As $\Omega(f, g)$ is derived from the system equations, while the input-output observability matrix depends on the recent sensor readings and inputs, if the system is observable the result of the above matrix multiplication can be expressed as a set of equations dependent on the known quantities of $y$ and $\underline{u}$. If the system is behaving in accordance with the nominal model $\mathcal{O}_{\triangle D} D(\underline{y}, \underline{u})$ will be as similar to $\mathcal{O}_{\triangle}(f, g)$ as measurement error and noise allow, and equation 4 will generate near-zero values $[15,16,22]$. However, if the system model has become inaccurate due to a fault changing the characteristics of the system, nonzero values will be generated, allowing the fault to be detected.

In fact, since the observability space by definition spans all that can be observed about the system using the current model, it can be shown that NLAR is guaranteed to react to any observable discrepancy $[15,16,22]$. The basis vectors of the observability space generated from system data (model $\left.\mathcal{O}_{\triangle D D}(y, \underline{u})\right)$ span a space dependent on the current behavior of the system that spans the observability space if the model is correct. The null-space of the model derived observability space $\Omega(\underline{f}, \underline{g})$ spans the space of information you shouldn't be able to see if the system is performing according to the model. Projecting the basis onto the null space instantly reduces a complex stream of sensor and input data into residual signals that show all the deviations from the expected model, and only those deviations.

NLAR test results can only be zero if the system equations are modeling the system behavior correctly. Any discrepancies, such as those that result from sensor noise, manifest as bias or noise in the NLAR output. However, given a good system model, most faults will cause deviations between the system and the model much greater than the difference caused by modeling inaccuracies. Faults will appear as large nonzero NLAR signals, thus allowing NLAR to be used as an effective tool for fault detection $[15,16,20,22]$.

The standard linear AR is shown to be a special case of NLAR in [16]. The linear technique described in [4] requires a linear system model like that described in equation 2. This can cause significant extra bias and noise in the linear AR tests if the system has nonlinear characteristics. NLAR can of course solve this problem.

\subsection{The NLAR Algorithm}

Although the full derivation of the NLAR method described above is too extensive to reproduce here, the following algorithm $[16,21,22]$ summarizes the necessary steps in deriving a suite of NLAR tests:

i. Determine the triangular nonlinear observability $\mathcal{O}_{\triangle}$ and its left null $\Omega$. 
ii. Determine the nonlinear dynamically derived observability $\mathcal{O}_{\triangle D D}$.

iii. Find the rank, $r_{j}$ (nonlin), of each observability sub-matrix in the observability "matrix" in $\nabla \mathcal{O}_{\triangle D D}$. Keep $r_{j}($ nonlin $)+1$ rows in each sub-vector. iv. Apply the NLAR equation to find the test residuals $\underline{R}: \Omega \mathcal{O} \triangle D D=\underline{R}$.

v. The number of independent NLAR tests, $N_{N L A R}$, can be determined by the equation $N_{N L A R}=\sum_{j=1}^{m}\left(r_{j}(\right.$ nonlin $\left.)\right)+(m-n)$. Use this equation to determine how many independent tests exist. Delete the redundant tests.

Full details of this method are discussed in $[16,21,22]$.

\subsection{Other Related Work}

Considerable work has been done using the concept of nonlinear observers in place of AR [3,13]. In fact, it has been shown that AR and observer based methods are equivalent in the linear case [24]. However, the proof in [24] is only applicable to linear systems, so the nonlinear observers are not necessarily the same as nonlinear AR. Additionally, the nonlinear observer based method [13] lacks the span guarantees of NLAR.

Zhirabok and Preobragenskaya have presented work with nonlinear AR test residuals based on observer theory [33]. Nonlinear test residuals are generated by following an algorithm for restating the model equations in terms of inputs $u_{i}$ and outputs $y_{i}$. Unfortunately this method does not use the observability to maintain the guarantees that make AR so desirable.

Wünnenberg and Frank have investigated methods for using dynamic thresholding with linear robotic AR test residuals to compensate for various modeling inaccuracies [11,32]. Instead of adapting the AR tests to the nonlinear systems, this work takes the practical approach of developing a system that runs in parallel to the AR system, predicts when the modeling inaccuracies will likely be large, and increases the thresholds on the AR residual tests appropriately.

Starosweicki and Comtet-Varga have produced some interesting work describing rigorous nonlinear AR limited classes of nonlinear systems [28,29]. This work discusses several methods of rigorously developing various ARlike test residuals without actually using the nonlinear observability. This work considers the spanning issue, but is limited by its neglect of the core observability issues of AR.

Isidori and De Persis have derived a geometric residual generator using nonlinear observability [7]. These residuals are similar in concept to those generated by AR in that they use the null space of the observability to test the system behavior. However, they are limited to checking for faults where both the disturbance and fault dynamics are known well enough to model accurately, making span guarantees much more troublesome $[12,25]$. This limits the Isidori approach to detecting well known and well modeled faults, while AR and NLAR are geared to detect all deviations from the model. 


\section{NLAR Applied to a Hydraulic System}

The Rosie mobile worksystem $[1,5,26]$ discussed in this section was the initial motivation for the study of nonlinear analytical redundancy. The nonlinearities introduced by hydraulic flow through components such as servovalves are a significant issue when dealing with hydraulic robots and systems $[2,6,14]$. These nonlinearities led us to investigate the application of modified AR fault detection techniques to deal in an approximate manner as shown in Appendix 9 with the nonlinear hydraulic system [18-20,23]. Eventually, we left the approximation limitations of our early techniques behind with increasingly rigorous notions of nonlinear analytical redundancy.

\subsection{The Rosie Mobile Worksystem}

The Rosie Mobile Worksystem is a tele-robotically operated, hydraulically driven robot, which provides locomotion and a four degree-of-freedom heavy manipulator arm which can be equipped with various tools and robot manipulators. Figure 1 is a photograph of the Rosie worksystem working at the CP-5 reactor. As described in the literature $[1,5,26]$, the robot consists of two main components or modules. The first module is a locomotor or mobile platform upon which is mounted the second module, a heavy manipulator. The locomotor module supports and transports the manipulator, and supplies it with power and control/communications. The locomotor consists of a central spine, or body core, upon which are attached front and rear drive wheel assemblies, an electronics enclosure, a hydraulic power supply system, a hydraulic enclosure for filters and valving, and a tether system. The locomotor platform is $198 \mathrm{~cm}$ wide, $107 \mathrm{~cm}$ high and $290 \mathrm{~cm}$ long (78 x $42 \times 114$ in.), supports an overall machine weight of $6,350 \mathrm{~kg}(14,000 \mathrm{lb}$.), and has a maximum speed of $0.6 \mathrm{~m} / \mathrm{s}(2.0 \mathrm{ft} / \mathrm{s})$.

Each wheel is powered individually by means of a geared, piston-type hydraulic motor, and is independently steered by means of a rotary actuator above that wheel. The front wheels are mounted on beams that can extend to provide additional stability when the manipulator arm is extended, as shown in Figure 2. The rear wheels are mounted on a pivoting beam for steering purposes.

The HPSS consists of a $45 \mathrm{~kW}(60 \mathrm{hp})$ supply which provides $114 \mathrm{l} / \mathrm{min}$. (30 GPM) at $20.7 \mathrm{MPa}(3,000 \mathrm{psi})$ for all robot operations. Electrical power and control are provided through a $61 \mathrm{~m}(200 \mathrm{ft})$ tether which is wound on a powered reel at the rear of the unit.

The heavy manipulator module supports and positions the tools that actually perform the decontamination and dismantlement (D\&D) functions. Shown with a fully-extended boom in Figure 2, the heavy manipulator performs the functions of waist rotation, shoulder pitch, outer forearm extension, inner forearm extension, and wrist pitch. The hardware to execute each of these functions is very similar and consists of a flow servovalve, an actuator, 


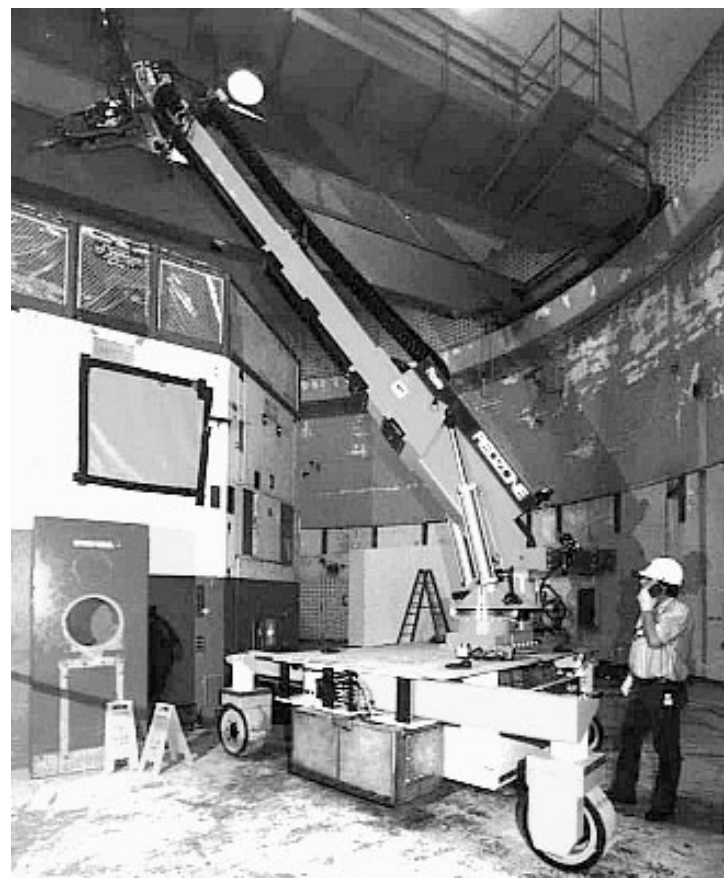

Fig. 1. The Rosie Mobile Worksystem at the CP-5 Reactor. (Courtesy of Sharon Curd at Oak Ridge National Labs)

and fluid components (tubing, fittings, etc.). The shoulder pitch and forearm extension functions have piston-cylinder actuators and the waist and wrist rotation are achieved through rotary actuators.

\subsection{The Hydraulic Testbed and Fault Simulation}

Failure modes, effects, and criticality analysis (FMECA) and fault tree based reliability analysis by researchers working with Rosie determined that the hydraulic wheel actuator subsystem was a vital component for the reliability of the mobile platform. A failure of a wheel mechanism might prevent the removal of the chassis from the reactor work site. This led to a project where the goal was to develop effective data analysis procedures (in our case AR-based fault detection) for hydraulic wheel actuators and then implement them on a testbed system located at Foster-Miller Inc., a company with considerable experience with hydraulic systems. The reliability of existing and future robots could then be enhanced by the results of this project. 


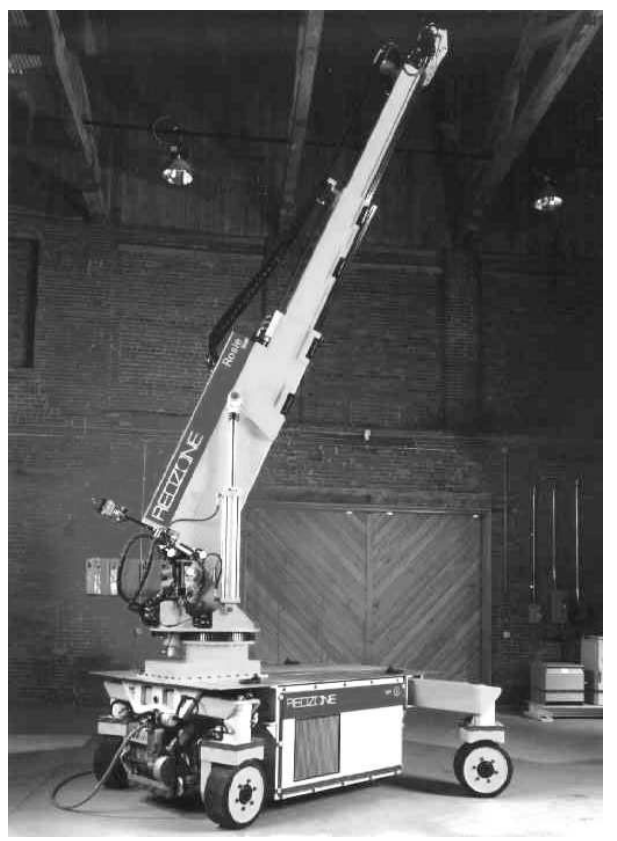

Fig. 2. Rosie with manipulator arm extended. (Courtesy of Sharon Curd at Oak Ridge National Labs)

The testbed was a hydraulic motor controlled by a hydraulic servovalve. (This was the main nonlinear component of the system.) The specific component selected for the test program was the hydraulic wheel motor from Rosie, a Black Bruin Model 404-080-2111 from Valmet Power Transmission Inc. It has a radial piston design and has a maximum power rating of $35 \mathrm{~kW}(47$ $\mathrm{hp}$ ), a maximum output speed of $185 \mathrm{rpm}$ and can deliver a torque of 2990 $\mathrm{Nm}$ at 250 bar (2205 ft-lb at $3600 \mathrm{psi}$ ). This motor was capable of driving a wheel directly and therefore accepting a substantial radial load, the exact value of load depending on the axial location of the load with respect to the motor.

The concept for the test rig itself is shown in Figures 3 and 4. A hydraulic motor powered by a HPSS was mounted on a machine bed. The output shaft was loaded radially by means of an adapter and a hydraulic jack assembly. Load was applied to the motor by means of an identical motor, used as a pump. The pump loading device was connected to the motor through a flexible coupling and differed from the motor in not having a hardened shaft and not having the "freewheeling with springs" option. The pump was fed 
through a separate hydraulic supply consisting of a low-pressure pump, cooler and reservoir. Load was controlled by means of a throttling valve, with a relief valve to prevent overpressure. Table 1 shows a listing of the motor-servovalve

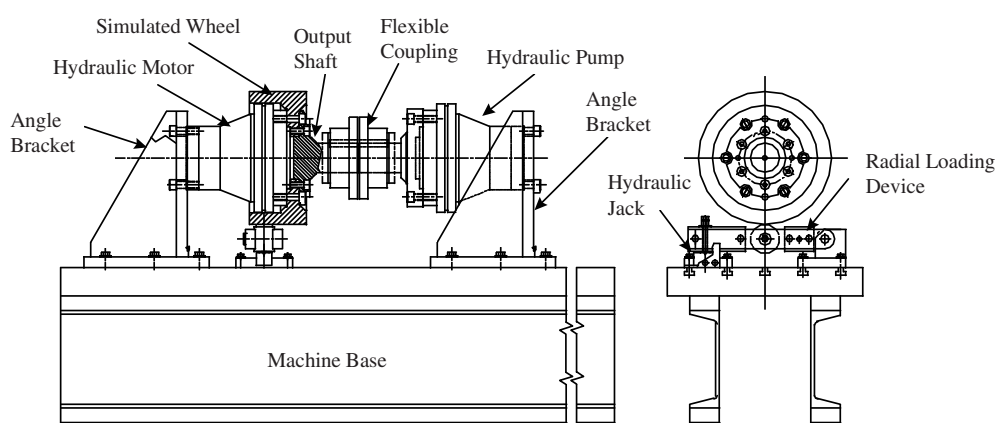

Fig. 3. Hydraulic test rig schematic. (Courtesy of Foster-Miller)

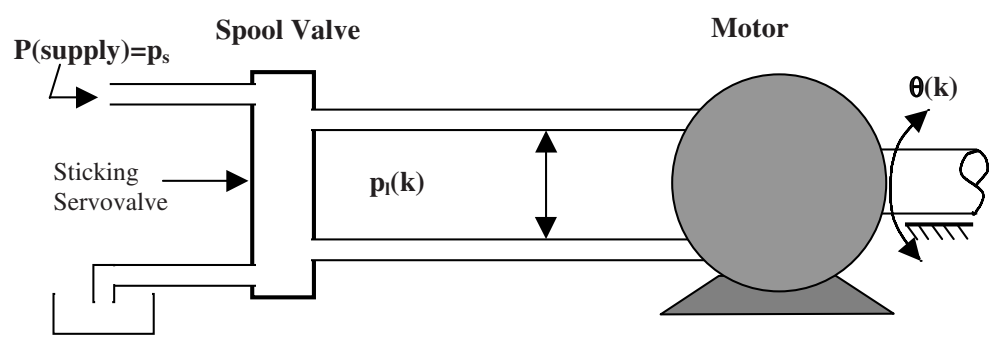

Fig. 4. Conceptual hydraulic system.

system faults investigated, along with the planned installation methods.

Problems with the servovalves focused on open windings and sticking of internal valve components. Open winding faults were simulated by inserting a relay in series with the winding. The relay was actuated by means of a bit output of the data acquisition board. This allowed a simple simulation of the fault in software. 
Table 1. Faults for the Foster-Miller hydraulic testbed.

\begin{tabular}{|l|l|l|}
\hline Component & Fault & Installation Method \\
\hline \hline Servovalve & Open winding & Relay \\
\hline Servovalve & Sticking valve & Change control profile in software \\
\hline Tachometer & Failed tachometer & Make input zero in controller \\
\hline Hydraulic motor & Ruptured line & Tee flow to separate tank \\
\hline
\end{tabular}

A sticking valve was simulated by altering the software control profile for the valve. For example, a new open loop control profile was substituted for the standard PID closed loop control algorithm. The new algorithm incorporated stick-slip behavior as needed to simulate the sticking valve.

Hydraulic systems are clearly vulnerable to many faults that electrical systems do not experience and are much harder to model due to their inherently nonlinear nature. However, a hydraulic system has considerable advantages as an actuator in a radioactive environment, as such systems are rugged and powerful, and much less likely to produce dangerous sparks than an electrical system. Therefore, it is sensible to use one and simply put some extra time and energy into ensuring the hydraulic system is adequately monitored.

\subsection{The Mathematical System Model}

Begin by defining the terms used in the model. Notation:

- $A, B$, and $C$ are the canonical discrete time state-space system matrices

- $B_{m}$ is the viscous damping coefficient

- $C_{t m}=c_{e m}+c_{i m}$ represent total, external, and internal leakage, respectively

- $d_{m}$ is the volumetric displacement of the motor

- $J_{t}$ is the inertia of the motor and load

- $K_{f}, k_{q}$ and $k_{c}$ are valve flow coefficients

- $M=k_{c}+C_{t m}$ is a generalized pressure coefficient

- $p_{l}$ and $p(k)$ are the (continuous and discrete) pressure drop across the motor as measured by the sensors

- $p_{s}$ is the hydraulic power supply; nominal pressure of 3000 PSI

- $Q$ is the net fluid flow into the spool valve

- $R_{1}$ through $R_{5}$ are nonlinear AR tests

- $t$ is the continuous time variable, $k$ the discrete time variable, $\Delta t$ is the time step

- $T_{g}$ is the torque generated by the motor

- $T_{l}$ is the load torque

- $u_{v}$ and $u(k)$ are the (continuous and discrete) servovalve positions

- $v_{t}$ is the volume of fluid within the motor

- $y(k)$ is the state vector 
- $\beta_{e}$ is the bulk modulus of the hydraulic fluid

- $\theta_{m}$ and $\theta(k)$ are the (continuous and discrete) position of the motor shaft - the derivative of this is measured by a velocity sensor

- $\rho$ is the hydraulic fluid density.

The following model equations are standard for hydraulic systems $[27,31]$ :

$$
\begin{aligned}
& T_{g}=p_{l} d_{m}=J_{t} \ddot{\theta}_{m}+B_{m} \dot{\theta}_{m}+T_{l}, \\
& Q=u_{v} K_{f} \sqrt{2\left(p_{s}-p_{l}\right) / \rho}=d_{m} \dot{\theta}_{m}+\left(c_{i m}+c_{e m}\right) p_{l}+\frac{v_{t} \dot{p}_{l}}{4 \beta_{e}}
\end{aligned}
$$

The state-space control model uses the following state vector:

$$
\underline{x}=\left[\begin{array}{c}
\theta \\
\dot{\theta} \\
p_{l}
\end{array}\right] .
$$

The second and third state variables are instrumented, but the first $(\theta)$ is not. The nonlinear control system formed by these assumptions is as follows:

$$
\begin{aligned}
& \underline{\dot{x}}=f(\underline{x})+g(\underline{x}) u,
\end{aligned}
$$

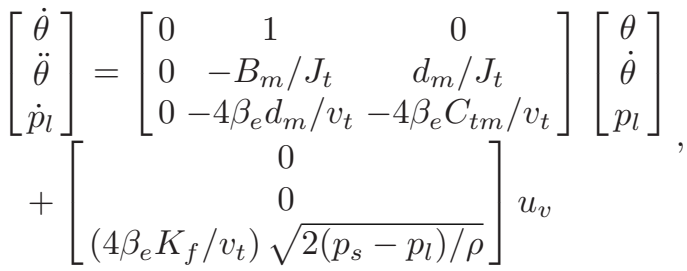

$$
\begin{aligned}
& \underline{y}=C \underline{x}, \quad C=\left[\begin{array}{lll}
0 & 1 & 0 \\
0 & 0 & 1
\end{array}\right], \quad \underline{y}=\left[\begin{array}{c}
\dot{\theta} \\
p_{l}
\end{array}\right]
\end{aligned}
$$

The intractability of nonlinear systems such as the one described above makes fault detection techniques like AR more important. The behavior of a nonlinear system is harder to predict and control, resulting in reduced safety and reliability, which in turn makes fault detection more prominent. However, model-based fault detection techniques require good models to be effective, and traditional linear AR is generally unsatisfactory for systems with significant nonlinear components. Figure 5 shows the modeling error for a standard linearization of the Rosie wheel actuator. As this error is not accounted for in linear AR, but is modeled in the NLAR method, the figure also shows the expected improvement in going from linear to nonlinear AR. 


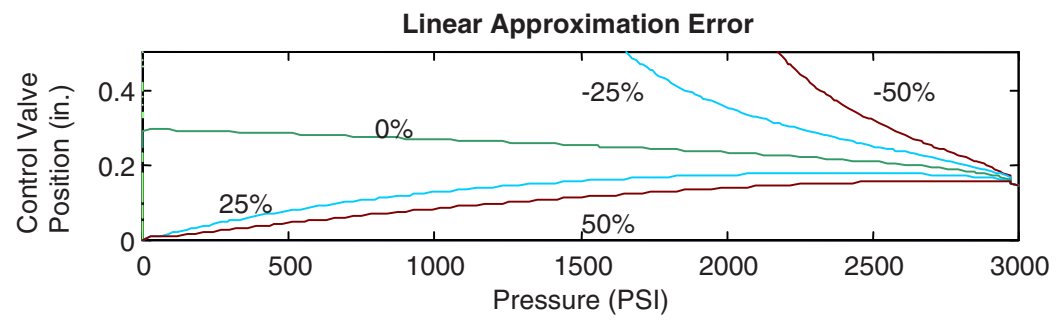

Fig. 5. Error due to linearization for hydraulic servovalve.

\section{Nonlinear Analytical Redundancy for the Rosie Wheel Actuator}

Now we apply our nonlinear analytical redundancy (NLAR) technique to the Rosie subsystem described above. This will allow us to avoid the mathematical errors caused by linearization, as shown in Figure 5. To begin, we calculate the grouped observability vector $\mathcal{O}_{\triangle}$ :

$$
\begin{aligned}
& \mathcal{O}_{\triangle}=\left[\begin{array}{c}
c_{1} \underline{x} \\
L_{f} c_{1} \underline{x}+L_{g} c_{1} \underline{x} u \\
L_{f f} c_{1} \underline{x}+L_{f g} c_{1} \underline{x} u+L_{g f} c_{1} \underline{x} u+L_{g g} c_{1} \underline{x} u^{2} \\
c_{2} \underline{x} \\
L_{f} c_{2} \underline{x}+L_{g} c_{2} \underline{x} u \\
L_{f f} c_{2} \underline{x}+L_{f g} c_{2} \underline{x} u+L_{g f} c_{2} \underline{x} u+L_{g g} c_{2} \underline{x} u^{2}
\end{array}\right], \\
& \mathcal{O}_{\triangle}=\left[\begin{array}{c}
\dot{\theta} \\
-B_{m} \dot{\theta} / J_{t}+d_{m} p_{l} / J_{t} \\
\left\{\begin{array}{c}
\left(B_{m}^{2} / J_{t}^{2}+-4 \beta_{e} d_{m}^{2} / J_{t} v_{t}\right) \dot{\theta} \\
+\left(-B_{m} d_{m} / J_{t}^{2}+-4 \beta_{e} d_{m} C_{t m} / J_{t} v_{t}\right) p_{l} \\
+\left(4 \beta_{e} d_{m} K_{f} / J_{t} v_{t}\right) \sqrt{2\left(p_{s}-p_{l}\right) / \rho} u \\
p_{l}
\end{array}\right\} \\
\left\{\begin{array}{c}
\left(-4 \beta_{e} d_{m} / v_{t}\right) \dot{\theta}+\left(-4 \beta_{e} C_{t m} / v_{t}\right) p_{l} \\
+\left(4 \beta_{e} K_{f} / v_{t}\right)\left(\sqrt{2\left(p_{s}-p_{l}\right) / \rho}\right) u
\end{array}\right\} \\
\left(\begin{array}{c}
4 B_{m} \beta_{e} d_{m} / J_{t} v_{t}+16 \beta_{e}^{2} d_{m} C_{t m} / v_{t}^{2} \\
+\left(8 \beta_{e}^{2} d_{m} K_{f} / v_{t}^{2}\right) \sqrt{2 / \rho\left(p_{s}-p_{l}\right)} u
\end{array}\right) \dot{\theta} \\
+\left(\begin{array}{c}
-4 \beta_{e} d_{m}^{2} / J_{t} v_{t}+16 \beta_{e}^{2} C_{t m}^{2} / v_{t}^{2} \\
+\left(8 \beta_{e}^{2} K_{f} C_{t m} / v_{t}^{2}\right) \sqrt{2 / \rho\left(p_{s}-p_{l}\right) u}
\end{array}\right) p_{l} \\
\left(-16 \beta_{e}^{2} K_{f} C_{t m} / v_{t}^{2}\right)\left(\sqrt{2\left(p_{s}-p_{l}\right) / \rho}\right) u \\
+\left(-16 \beta_{e}^{2} K_{f}^{2} / \rho v_{t}^{2}\right) u^{2}
\end{array}\right\} .
\end{aligned}
$$


Note the nonlinear sub-observability matrices $c_{i} \nabla \mathcal{O}_{\triangle}$ have no terms containing $\theta$, so the system is rank two and only three terms are needed in each $C_{j}$. This also means four independent NLAR tests are expected.

Now calculate the null-matrix $\Omega$ :

$$
\Omega \mathcal{O}_{\triangle}=\left[\begin{array}{cccccc}
\Omega_{11} & \dot{\theta} & 0 & 0 & 0 & 0 \\
\Omega_{21} & 0 & \dot{\theta} & 0 & 0 & 0 \\
\Omega_{31} & 0 & 0 & \dot{\theta} & 0 & 0 \\
\Omega_{41} & 0 & 0 & 0 & \dot{\theta} & 0 \\
\Omega_{51} & 0 & 0 & 0 & 0 & \dot{\theta}
\end{array}\right] \mathcal{O}_{\triangle}=0,
$$

where:

$$
\Omega_{11}=\frac{B_{m}}{J_{t}} \dot{\theta}+\frac{-d_{m}}{J_{t}} p_{l},
$$

and

$$
\begin{aligned}
\Omega_{12}= & \left(\frac{-B_{m}^{2}}{J_{t}^{2}}+\frac{4 \beta_{e} d_{m}^{2}}{J_{t} v_{t}}\right) \dot{\theta}+\left(\frac{B_{m} d_{m}}{J_{t}^{2}}+\frac{4 \beta_{e} d_{m} C_{t m}}{J_{t} v_{t}}\right) p_{l}, \\
& +\left(\frac{-4 \beta_{e} d_{m} K_{f}}{J_{t} v_{t}} \sqrt{2\left(p_{s}-p_{l}\right) / \rho}\right) u \\
\Omega_{13}= & -p_{l}, \\
\Omega_{14}= & 4 \beta_{e} d_{m} / v_{t} \dot{\theta}+4 \beta_{e} C_{t m} / v_{t} p_{l} \\
& +\left(-4 \beta_{e} K_{f} / v_{t}\right) \sqrt{2\left(p_{s}-p_{l}\right) / \rho} u \\
& \left(\begin{array}{l}
-4 B_{m} \beta_{e} d_{m} / J_{t} v_{t}-16 \beta_{e}^{2} d_{m} C_{t m} / v_{t}^{2} \\
+\left(-8 \beta_{e}^{2} d_{m} K_{f} / v_{t}^{2}\right) \sqrt{2 / \rho\left(p_{s}-p_{l}\right) u}
\end{array}\right) \dot{\theta} \\
\Omega_{51}= & +\left(\begin{array}{l}
4 \beta_{e} d_{m}^{2} / J_{t} v_{t}-16 \beta_{e}^{2} C_{t m}^{2} / v_{t}^{2}+ \\
\left(-8 \beta_{e}^{2} K_{f} C_{t m} / v_{t}^{2}\right) \sqrt{2 / \rho\left(p_{s}-p_{l}\right) u}
\end{array}\right) p_{l} \\
& +\left(16 \beta_{e}^{2} K_{f} C_{t m} / v_{t}^{2}\right) \sqrt{2\left(p_{s}-p_{l}\right) / \rho} u+16 \beta_{e}^{2} K_{f}^{2} / \rho v_{t}^{2} u^{2}
\end{aligned}
$$

Then calculate $\mathcal{O}_{\triangle D D}$ :

$$
\mathcal{O}_{\triangle D D}=\left[\begin{array}{c}
y_{1}(t) \\
\dot{y}_{1}(t) \\
\ddot{y}_{1}(t) \\
y_{2}(t) \\
\dot{y}_{2}(t) \\
\ddot{y}_{2}(t)-\left(4 \beta_{e} K_{f} / v_{t}\right) \sqrt{2\left(p_{s}-y_{2}(t)\right) / \rho} \dot{u}(t)
\end{array}\right] .
$$

Then simply apply the NLAR equation:

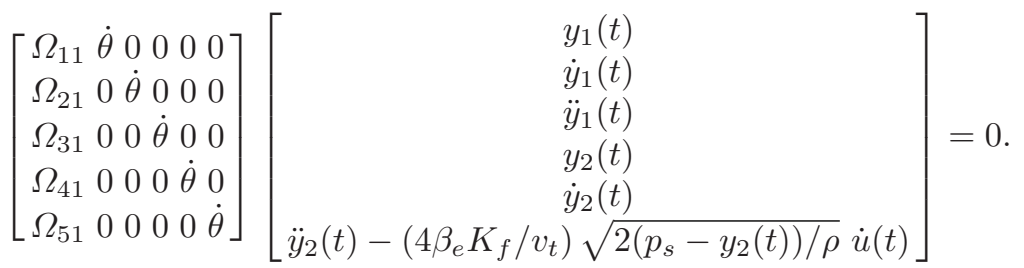


to get the NLAR tests.

$$
\begin{aligned}
& R_{1}=-\dot{y}_{1}(t)-\frac{B_{m}}{J_{t}} y_{1}(t)+\frac{d_{m}}{J_{t}} y_{2}(t) \\
& \begin{aligned}
R_{2}=\begin{array}{l}
-\ddot{y}_{1}(t)+\left(B_{m}^{2} / J_{t}^{2}-4 \beta_{e} d_{m}^{2} / J_{t} v_{t}\right) y_{1}(t) \\
+\left(-B_{m} d_{m} / J_{t}^{2}-4 \beta_{e} d_{m} C_{t m} / J_{t} v_{t}\right) y_{2}(t)
\end{array}
\end{aligned} \\
& R_{3}=-y_{2}(t) y_{1}(t)+y_{1}(t) y_{2}(t) \\
& \begin{aligned}
R_{4}= & -\dot{y}_{2}(t)-4 \beta_{e} d_{m} y_{1}(t) / v_{t}-4 \beta_{e} C_{t m} y_{2}(t) / v_{t} \\
& +\left(4 \beta_{e} K_{f} / v_{t}\right) \sqrt{2\left(p_{s}-y_{2}(t)\right) / \rho} u
\end{aligned} \\
& -\ddot{y}_{2}(t) \\
& +\left(\begin{array}{c}
4 B_{m} \beta_{e} d_{m} / J_{t} v_{t}+16 \beta_{e}^{2} d_{m} C_{t m} / v_{t}^{2} \\
+\left(8 \beta_{e}^{2} d_{m} K_{f} / v_{t}^{2}\right) \sqrt{2\left(p_{s}-y_{2}(t)\right) / \rho} u
\end{array}\right) y_{1}(t) \\
& R_{5}=+\left(\begin{array}{l}
-4 \beta_{e} d_{m}^{2} / J_{t} v_{t}+16 \beta_{e}^{2} C_{t m}^{2} / v_{t}^{2} \\
+\left(8 \beta_{e}^{2} K_{f} C_{t m} / v_{t}^{2}\right) \sqrt{2\left(p_{s}-y_{2}(t)\right) / \rho} u
\end{array}\right) y_{2}(t) \\
& -\left(16 \beta_{e}^{2} K_{f} C_{t m} / v_{t}^{2}\right) \sqrt{2\left(p_{s}-y_{2}(t)\right) / \rho} u-16 \beta_{e}^{2} K_{f}^{2} u^{2} / \rho v_{t}^{2} \\
& +\left(4 \beta_{e} K_{f} / v_{t}\right) \sqrt{2\left(p_{s}-y_{2}(t)\right) / \rho} \dot{u}
\end{aligned}
$$

Note that $R_{3}$ is trivial, so the expected number of independent NLAR tests is generated. $R_{1}$ and $R_{4}$ correspond to the model equations. $R_{2}$ and $R_{5}$ correspond to the convolved first derivatives of the model equations. This is a common result of AR and NLAR analyses, and a reassuring one. The tests generated relate directly and intuitively to the system being analyzed.

\section{Results}

The following results were generated by applying nonlinear analytical redundancy techniques to recorded experimental data from the hydraulic testbed described previously. In the case of the Rosie wheel actuator, the NNAR ${ }^{1}$ tests discussed in Appendix 9 and in previous papers [18-20,23] are the same

\footnotetext{
${ }^{1}$ It turns out that the spans of linear and nonlinear observability spaces for this sytem are similar enough that previously derived approximate techniques [19,23], created by us to allow application of linear AR to nonlinear systems, were correct for this particular system. (This is something of a co-incidence, and not true for a generaleralized nonlinear system.) For this specific case the approximation used in the NNAR technique is exact. The nearly nonlinear AR (NNAR) technique
} 
as the NLAR tests. While this is a useful coincidence validating previously derived approximate test results, the most important aspect of the following results remains that they validate the NLAR technique using physical testbed data.

Each of the following result sections shows all four of the NLAR test residuals for both a baseline data run (light grey) and a run with an injected fault (black). As NLAR test residual magnitudes are a somewhat arbitrary function of basis choice during derivation, it is important to note that the magitude of the plot along the y-axis is important only in comparison with other runs. It is thus the shape and magnitude of the residual relative to the baseline test run that is indicative of faulty or fault-free conditions.

The hydraulic engine speed and the load on the system (in terms of PSI across the load pump) are shown with each set of residuals.

\subsection{Servovalve, Open Winding Fault}

This fault was modeled by physically opening the winding on the hydraulic servovalve and inserting a relay to short circuit the control solenoid. The fault is detected easily by NLAR -the onset and duration of this fault is clear in all AR tests - large spikes in two of the NLAR test residuals $\left(R_{1}\right.$ and $\left.R_{5}\right)$ show the fault the instant after it appears. Large steps in the other two tests are almost as fast. This is because the open winding acts like a large step input that is not accounted for in the model, degrading the accuracy of the model greatly in a short time period. This naturally provokes a strong AR response, which allows us to determine the system is operating under faulty conditions.

\subsection{Sticking Wheel Motor Servovalve Fault}

In the simulation of this fault, the system was ramped from zero speed to a speed of 5 RPM (equal to the fault free run) while the control input was intermittantly set to zero to simulate sticking. This fault is evident on all of the AR tests, although $R_{1}$ and $R_{5}$ show the clearest results. The system starts out close to the model but rapidly departs from model-following behavior if the control system tries to apply a nonzero control input while the servovalve is 'stuck.' This causes the spikes in the NLAR tests shown in Figure 5.2 that make the fault easily detectable.

is an approximate but useful method developed early in the course of this work to deal with the nonlinearities of the Rosie hydraulic actuator [18-20,23]. This method is not truly nonlinear in nature, however, as it uses the linearized system model to derive the AR tests. (The approximate NNAR and PLAR methods are briefly described in Appendix 9.) 

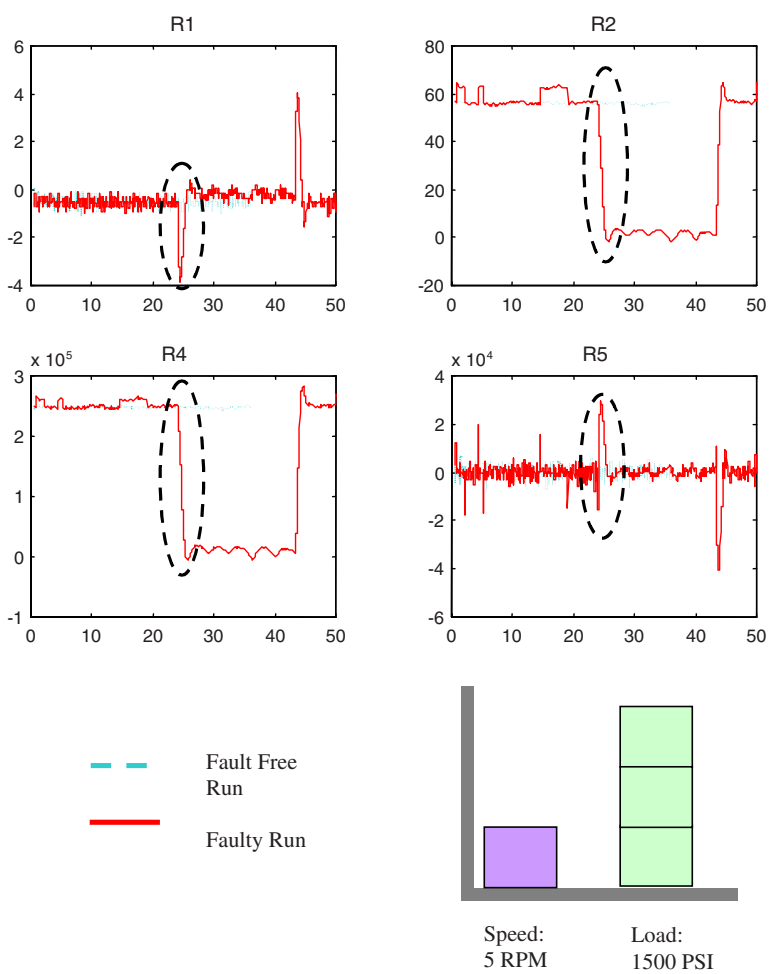

Fig. 6. Servovalve, open winding fault run.

\subsection{Loss of Speed Feedback (Tachometer) Fault}

The failed sensor invalidates the control loop if the commanded velocity is nonzero. The sensor reports zero angular velocity, so the controller tries to increase the speed by providing more power, quickly leading to a runaway system. (The testbed had a limiting device to prevent damage when this happened.) NLAR detects this as a deviation from the model-expected behavior almost instantly - the control inputs and sensor values disagree. This leads to the clear error signals seen in the figure.

\section{The Importance of Model Accuracy}

It bears repeating that the success of the NLAR fault detection technique is limited by the accuracy of the system model. The successful tests described 

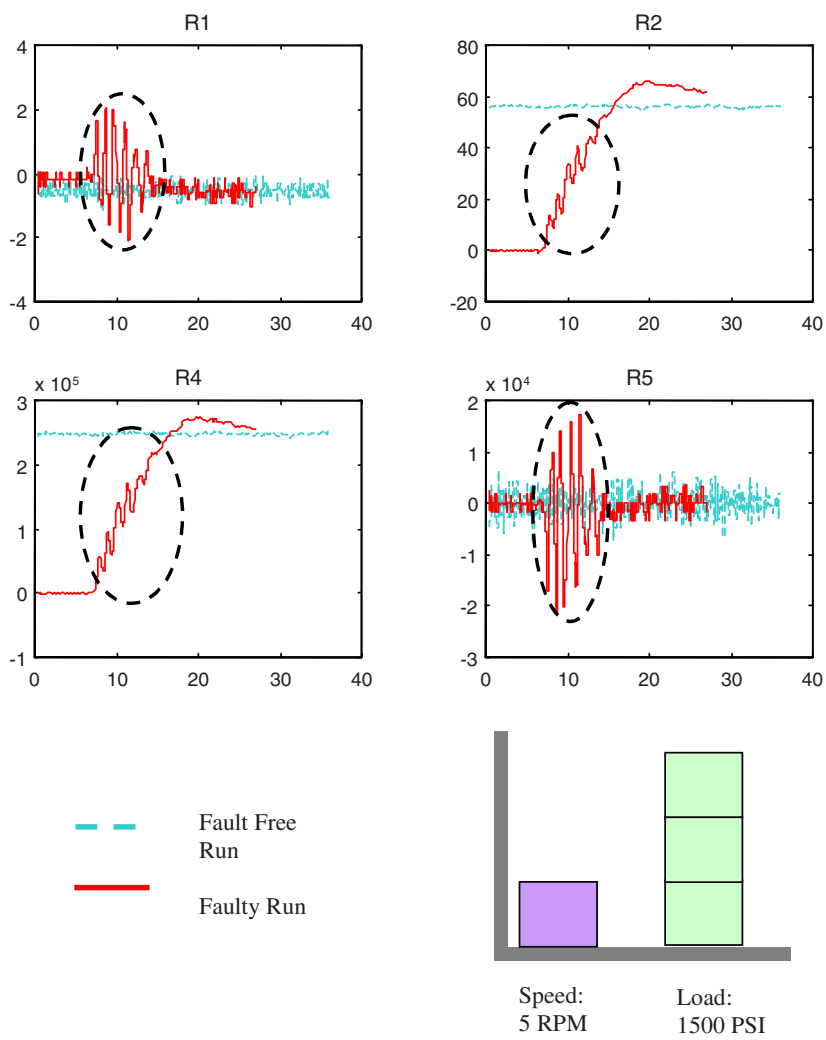

Fig. 7. Sticking wheel motor servovalve fault run.

in Section 5 are notable in that they are detecting faults that cause large and immediate deviations from the system model described in Section 3.3. All faults are not so tractable. Consider the following fault, which represents a leak in the motor-valve system, shown in figure 9 .

Why does NLAR not generate a clear and unambiguous signal as it did for the other three faults? Investigation shows that as NLAR is a model based fault detection algorithm, it is limited by the accuracy of the model. The NLAR test residuals shown were applied as a post-analysis to an incompletely documented system. While the model presented in Section 3.3 is a good mathematical representation of this hydraulic system, several of the constants in the system are only approximately determined or not as constant as one might desire. For example, the supply pressure $p_{s}$ is approximated as 

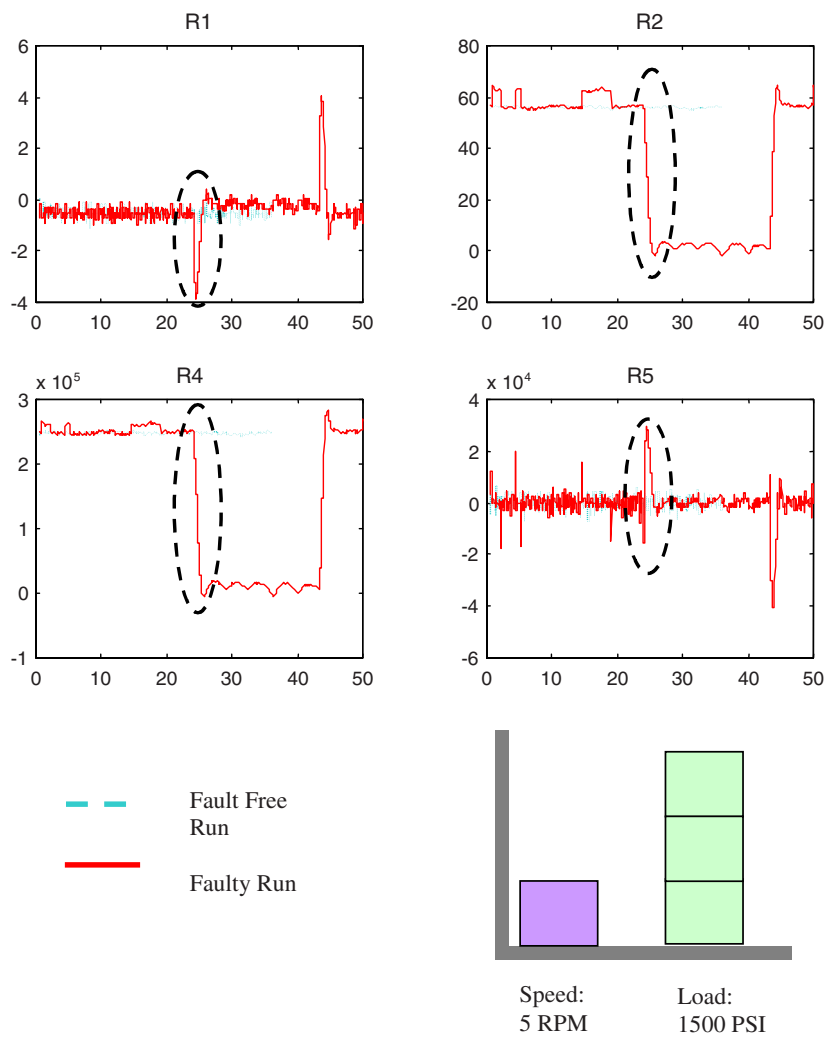

Fig. 8. Loss of speed feedback (tachometer) fault run.

a constant 3000 PSI. This is a rough approximation made necessary by the lack of a pressure sensor to give this value directly. Similarly, many of the parameters describing the liquid behavior of the hydraulic fluid are actually functions of the oil temperature. Several other constants are catalog values of uncertain accuracy. For most error based control applications, these approximations are acceptable. However, for model based fault detection, every inaccuracy limits the resolution of the tests. In the case of the leak fault shown, the resolution of the NLAR tests is too coarse to detect the tiny error caused by the steady leakage of a small percentage of the hydraulic fluid. We are confident that better modeling and proper instrumentation of the system could overcome this and make NLAR capable of detecting quite such leak faults. For many sensitive systems, better modeling is a small price to pay for improved reliability, and NLAR is a good choice for such systems. 

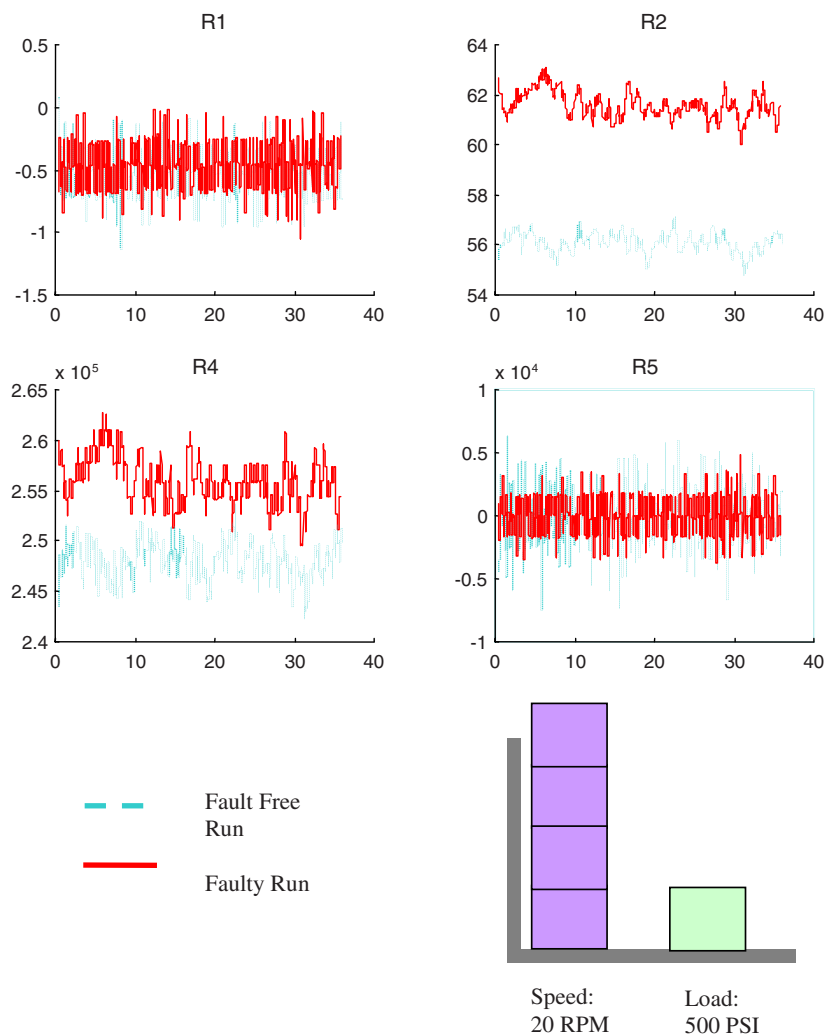

Fig. 9. Leak in motor-valve system fault.

\section{Conclusions}

Analytical redundancy is a model based fault detection technique, and thus requires an accurate system model to be effective. Previous AR theory has been limited to linear system models; this is a serious problem in a nonlinear world. Advances in nonlinear control theory have opened the door to the nonlinear realm. This allows us to apply the AR method rigorously to most nonlinear systems without losing any of the valuable theoretical assurances of complete coverage inherent to the AR method. The Rice and Clemson group has developed such theoretically robust nonlinear AR techniques.

Nonlinear AR fault detection is a useful monitoring method for hydraulic systems such as Rosie that must operate in hazardous environments. Better fault detection for hydraulics will reduce the costs associated with failures of 
such systems in the workplace by minimizing damage done by and to faulty systems as well as the amount of time wasted by false alarms. Safety and reliability are critical for success of many operations in hazardous environments, and this work represents the first detailed examination of nonlinear AR fault detection for these types of hydraulic robot systems.

\section{Acknowledgements:}

This work was supported in part by the National Science Foundation under grants IRI-9526363 and CMS 9796328, by DOE Sandia National Laboratory Contract \#AL3017, NASA grant NAG5-9785, NSF/EPSCoR grant EPS-9630167, and DOE contract DE-FG07-97ER 14830. The authors would also like to thank V. Jammu, R. W. Gamache, M. Martin, T. Walter, and D. McCauley at Foster-Miller Technologies, Albany NY

\section{Appendix: Approximate Approaches to NLAR}

One way to deal with a system model that is nonlinear enough to change considerably as it moves through the workspace is to create several sets of AR tests for the system linearized about state vectors located in each region of interest. In the local region each set would be more accurate than a general linearization of the control equations for the entire workspace. An example of this technique, and the improvement it brings, is illustrated below in Figure 10. In this case, generated for the hydraulic servovalve system, the pressurevalve position workspace in which the flow equation is nonlinear is divided up into nine equal regions. The model equation is linearized about a point at the center of each and normal AR tests are derived. (Due to the symmetry of the system, only four linearizations are needed in practice.) During operation, the AR test used is the one that was linearized about a point closest to the current position, with interpolated transitions near the borders of each region.

Figure 11 shows the results from a fault free run and a faulty run (the fault was a large leak) of PLAR tests on a simulated hydraulic servovalve $[18,19]$. The fault free run shows how PLAR minimizes the drifting away from the model errors of pure linear AR. Before the test residual can drift far from the point about which it was linearized, the system transitions into another, more appropriate AR test linearized about a point closer to the actual state of the system, leading to a saw-toothed residual about the nominally correct zero value. The results in a test run with a large leak fault added to the hydraulic system show that this saw-tooth is about an order of magnitude smaller than the fault signature.

Nearly nonlinear analytical redundancy is a natural outgrowth of PLAR. Although each PLAR partition uses a different linearization of the control equations, the different linearizations all share the same basic form - they 


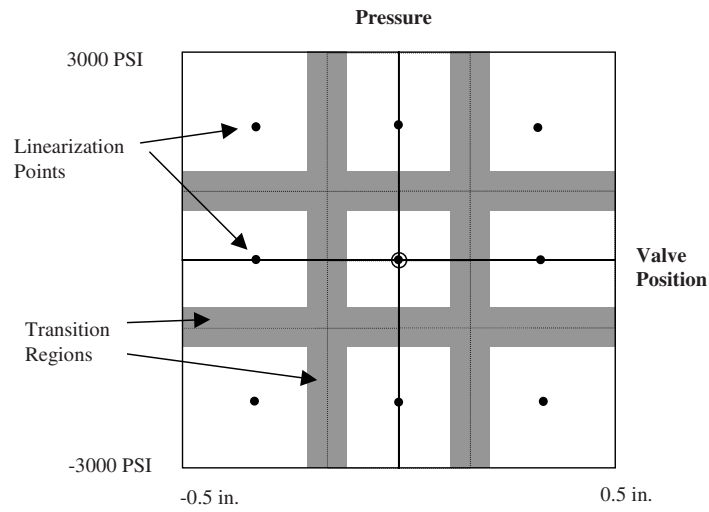

Fig. 10. PLAR division of hydraulic servovalve workspace.
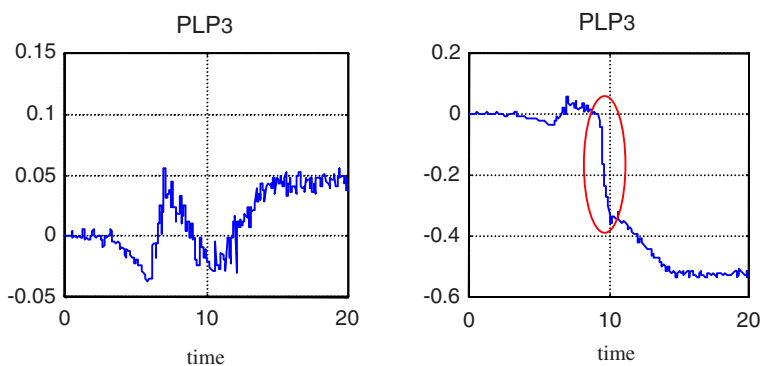

Fig. 11. PLAR fault free (left) and faulty (right) test results for simulated hydraulic servovalve.

are tangents to the control system at the point of linearization. This means that the AR tests generated by the different linearizations will be, in essence, tangents to some more accurate nonlinear AR test. It is reasonable to approach this test by dividing the workspace into many closely packed regions and taking appropriate linearizations. In the limit of infinitely small regions, this is a nonlinear AR test! However, there is a much simpler method of finding these tests. Recall that AR tests tend to be combinations of the model equations, sensor comparisons, and their derivatives. By performing linear $\mathrm{AR}$ on a linearized system and identifying the relationship between the AR residual tests and the control model and sensors, it is possible to find nonlin- 
ear AR tests by simply duplicating this relationship to the nonlinear control system and sensors! For example, if the AR tests for the linear system are the linearized model equations, their first derivatives, and direct sensor comparisons between the linearized sensors, the nonlinear model equations, their first derivatives, and comparisons of the nonlinear sensors can be used as NNAR tests.

Why, then, are these tests referred to as merely "nearly nonlinear" rather than "fully nonlinear?" Unfortunately, as they use the linear observability matrix they are still using linear approximations of the observability nullspace, $\Omega$. It can be shown that this is different than the nonlinear null-space; in fact the two can have a different rank. The AR guarantees of testing for all of the possible model deviations efficiently are thus not valid in PLAR and NNAR. Any AR method that doesn't use the full nonlinear observability space will suffer from this drawback. This is what makes fully nonlinear AR using the nonlinear observability space so desirable, and relegates PLAR and NNAR to secondary roles. These methods do not require extensive nonlinear calculation to use, but they are essentially approximations of the complete NLAR technique.

\section{References}

1. Bares L. C., Conley L.S., Thompson B.R. (1997) Rosie: A Mobile Worksystem for D \& D: Overview of System Capabilities and CP-5 Reactor Application. Proc. of ANS 7th Topical Meeting on Robotics and Remote Systems, Augusta, GA, 471-477

2. Bu F., Yao B. (2001) Nonlinear Model Based Coordinated Adaptive Robust Control of Electro-hydraulic Robotic Arms via Overparametrizing Method. IEEE International Conference on Robotics and Automation, Seoul, Korea

3. Caccavale F., Walker I.D. (1997) Observer-Based Fault Detection for Robot Manipulators. IEEE Int. Conf. on Robotics and Automation. Albuquerque, NM, 2881-2887

4. Chow E.Y., Willsky A.S. (1984) Analytical Redundancy and the Design of Robust Failure Detection Systems. IEEE Trans. on Automatic Control 29:603614

5. Conley L., Hamel W.R., Thompson B.R. (1995) Rosie: A Mobile Worksystem for Decontamination and Dismantlement Operations. Proc. of the ANS 6th Topical Meeting on Robotics and Remote Systems, Monterey, CA, 231-238

6. Daachi B., Benallegue A., M'Sirdi N. K. (2001) A Stable Neural Adaptive Force Controller for a Hydraulic Actuator. IEEE Int. Conf. on Robotics and Automation, Seoul, Korea

7. De Parsis C., Isidori A. (2001) A Geometric Approach to Nonlinear Fault Detection and Isolation. IEEE Trans. on Automatic Control 46:853-865

8. Department of Energy, Washington, D.C. (1993) Environmental Restoration and Waste Management 5-Year Plan, Fiscal Years 1994-1998. DOE/S-00097P, Vol. 1-2 
9. Department of Energy, Federal Energy Technology Center, Morgantown, WV (1997) Environmental Waste Management Project Fact Sheet. http://www.fetc.doe.gov/publications/factsheets/ewm/index.html

10. Dhillon B.S. (1991) Robot Reliability and Safety, Springer-Verlag, New York, NY

11. Ding X., Frank P.M. (1991) Frequency Domain Approach and Threshold Selector for Robust Model-Based Fault Detection and Isolation. Proc. of IFAC Fault Detection, Supervision and Safety for Technical Processes, Baden-Baden, Germany, 271-276

12. Ding X., Guo L., Jeinsch T. (1999) A Characterization of Parity Space and Its Application to Robust Fault Detection. IEEE Transactions on Automatic Control 44:337-343

13. Hammouri H., Kinnaert M., El Yaagoubi E.H. (1999) Observer-Based Approach to Fault Detection and Isolation for Nonlinear Systems. IEEE Transactions on Automatic Control 44:1879-1884

14. Honegger M., Corke P. (2001) Model-Based Control of Hydraulically Actuated Manipulators. IEEE International Conference on Robotics and Automation, Seoul, Korea

15. Isidori A. (1995) Nonlinear Control Systems. Springer-Verlag, London,UK

16. Leuschen M.L. (2001) Derivation and Application of Nonlinear Analytical Redundancy Techniques with Applications to Robotics. PhD thesis, Department of Electrical and Computer Engineering, Rice University, Houston, TX

17. Leuschen M.L., Walker I.D., Cavallaro J.R. (1998) Robot Reliability Through Fuzzy Markov Models. Proc. IEEE Annual Reliability and Maintainability Symposium, Anaheim, CA, 209-214

18. Leuschen M.L., Walker I.D., Cavallaro J.R. (1999) Investigation of Reliability for Hydraulic Robots Using Analytical Redundancy. Proc. IEEE Annual Reliability and Maintainability Symposium, Washington, DC, 122-128

19. Leuschen M.L., Walker I.D., Cavallaro J.R. (1999) Monitoring and Diagnostics for a Hydraulic Robot in Hazardous Environments. Proc. of ANS 8th Topical Meeting on Robotics \& Remote Systems, Pittsburgh, PA

20. Leuschen M.L., Walker I.D., Cavallaro J.R. (2001) Experimental AR Fault Detection Methods for a Hydraulic Robot. Proc. of ANS 9th Topical Meeting on Robotics and Remote Systems, Seattle, WA, F-131

21. Leuschen M.L., Walker I.D., Cavallaro J.R. (2002) Robotic Fault Detection Using Nonlinear Analytical Redundancy. IEEE International Conference on Robotics and Automation, Washington, DC

22. Leuschen M.L., Walker I.D., Cavallaro J.R. (2002) Nonlinear Analytical Redundancy for Fault Detection. Submitted to: IEEE Trans. on Automatic Control

23. Leuschen M.L., Walker I.D., Cavallaro J.D., Gamache R.W., Martin M. (2000) Experimental AR Fault Detection Methods for a Hydraulic Robot. Proc. of 18th International Systems Safety Conference, Fort Worth, TX, 402-409

24. Magni J.F., Mouyon P. (1994) On Residual Generation by Observer and Parity Space Approaches. IEEE Trans. on Automatic Control 39:441-447

25. Nyberg M., Nielsen L. (2000) A Universal Chow-Willsky Scheme and Detectability Criteria. IEEE Trans. on Automatic Control 45:152-156

26. RedZone Robotics Inc. The Rosie Mobile Worksystem. http://www.redzone.com/downloads/Rosie.pdf.

27. Stadler W. (1995) Analytical Robotics and Mechatronics. Mc-Graw-Hill, Inc., New York, NY 
28. Staroswiecki M., Cassar J.P, Comtet-Varga G. (1997) Analytic Redundancy Relations for State Affine Systems. Proc. of 1997 Fourth European Control Conference, Brussels, Belgium

29. Staroswiecki M., Comtet-Varga G. (2001) Analytical Redundancy Relations for Fault Detection and Isolation in Algebraic Dynamic Systems. Automatica 37:687-699

30. Visinsky M.L., Cavallaro J.R., Walker I.D. (1995) A Dynamic Fault Tolerance Framework for Remote Robots. IEEE Transactions on Robotics and Automation 11:477-490

31. White F.M. (1994) Fluid Mechanics. McGraw-Hill, Inc., USA

32. Wünnenberg J., Frank P.M. (1990) Dynamic Model Based Incipient Fault Detection Concept for Robots. Proc. of 11th IFAC World Congress, Tallinn, Estonia, 61-66

33. Zhirabok A.N., Preobragenskaya O.V. (1993) Instrument Fault Detection in Nonlinear Dynamic Systems. Proc. of 1993 IEEE International Conference on Systems, Man, and Cybernetics, Le Touquet, France, 114-119 